\begin{tabular}{|c|l|}
\hline Title & Dynamic transitions through scattors in dissi pative sy stems \\
\hline Author(s) & Nishiura, Y asumasa; Teramoto, Takashi; Ueda, Kei-ichi \\
\hline Citation & $\begin{array}{l}\text { Chaos: An Interdisciplinary Journal of Nonlinear Science, 13(3), 962-972 } \\
\text { https:/doi.org/10.1063/1592131 }\end{array}$ \\
\hline Issue Date & 2003-09 \\
\hline Doc URL & http://hdl.handle.net/2115/35227 \\
\hline Rights & ○ 2003 A merican Institute of Physics \\
\hline Type & article \\
\hline File Information & nishiura 33.pdf \\
\hline
\end{tabular}

Instructions for use 


\title{
Dynamic transitions through scattors in dissipative systems
}

\author{
Yasumasa Nishiuraa) \\ Research Institute for Electronic Science, Hokkaido University, Sapporo 060-0812, Japan \\ Takashi Teramoto and Kei-Ichi Ueda \\ Meme Media Laboratory, Hokkaido University, Sapporo 060-0813, Japan
}

(Received 20 February 2003; accepted 19 May 2003; published 22 August 2003)

\begin{abstract}
Scattering of particle-like patterns in dissipative systems is studied, especially we focus on the issue how the input-output relation is controlled at a head-on collision where traveling pulses or spots interact strongly. It remains an open problem due to the large deformation of patterns at a colliding point. We found that a special type of unstable steady or time-periodic solutions called scattors and their stable and unstable manifolds direct the traffic flow of orbits. Such scattors are in general highly unstable even in the one-dimensional case which causes a variety of input-output relations through the scattering process. We illustrate the ubiquity of scattors by using the complex Ginzburg-Landau equation, the Gray-Scott model, and a three-component reaction diffusion model arising in gas-discharge phenomena. (C) 2003 American Institute of Physics.
\end{abstract}

[DOI: $10.1063 / 1.1592131]$

Collision process of particle-like patterns in dissipative systems is studied. A variety of input-output relations such as annihilation, repulsion, fusion, and even chaotic dynamics is observed after collision, which makes a sharp contrast with that of integrable systems. Unstable stationary or time-periodic patterns called scattors direct a traffic flow of orbits during the collision process in the infinite-dimensional space along their stable and unstable manifolds. Local dynamics near the scattor and connections among scattors form an underlying mechanism to predict the output. We illustrate this for several representative systems including the complex Ginzburg-Landau model, the Gray-Scott model, and a three-component reaction-diffusion model arising in gas-discharge phenomenon.

\section{INTRODUCTION}

Spatially localized objects such as pulses and spots form a representative class of dynamic patterns in dissipative systems. One of the recent remarkable discoveries is a variety of interacting manners among those patterns, which makes a sharp contrast with well-known annihilation of excitable waves like the pulses of the FitzHugh-Nagumo equations. Bouncing like elastic objects upon collision or merging into a single spot is the tip of the iceberg. Such a phenomenon has been observed experimentally and numerically, for instance, in gas-discharged system, ${ }^{1,2}$ CO-oxidization process, ${ }^{3-5}$ chemical reactions, ${ }^{6-13}$ and reaction-diffusion systems with a global feedback system. ${ }^{14,15}$ Suppose there are spatially localized moving patterns such as pulses or spots in a free space. A qualitative change for the pattern may occur either in interaction with other patterns through collision or intrinsic instability such as splitting or destruc-

\footnotetext{
${ }^{a)}$ Electronic mail: nishiura@aurora.es.hokudai.ac.jp
}

tion by itself. It is known that if a localized pattern has an intrinsic instability like self-replication, then combined with a self-destruction or annihilation process, it produces, in general, a complicated dynamics like spatiotemporal chaos ${ }^{16}$ or Sierpinski gaskets. ${ }^{17}$ In order to understand the whole dynamics of such complex patterns, a computer-aided geometric approach is quite useful as was shown by Refs. 16, 18, and 19. On the other hand, if a traveling pattern is asymptotically stable, it persists within a weak interaction regime, ${ }^{10,11}$ therefore any qualitative change should occur to such a pattern only through strong interaction with other moving patterns, however the underlying mechanism to control such a process is very little known partly because it is a large deformation in infinite dimensional space. The aim of this paper is to present a new viewpoint to clarify the process of strong interaction. In particular, we focus on head-on collisions among traveling patterns. Our approach to scattering phenomena is to find an origin of the sorting mechanism rather than to try to describe the details of large deformation of the solution. It turns out that such an origin is identified as the stable and unstable directions of a special type of unstable solutions called scattors, which link input to output at collision. A closely related work pointing out the importance of such saddles is Ref. 20, which analyzed the transition from annihilation to preservation of colliding waves arising in a simple model of continuum of pendula subjected to a constant torque and a viscous damping.

Let us look at a typical strong collision process like Fig. 1 (see Sec. IV for details) where the input is two incoming pulses and the output is just one outgoing pulse. Natural questions are how such a large deformation is controlled and how we can predict the output from the input. In Fig. 1 two scattors (see twin-horn and fused patterns in Sec. IV) play a pivotal role in understanding the scattering process. In fact the orbital behaviors are guided by the stable and unstable manifolds of the scattors, and the output can be classified by looking at the outcome from the scattor. In other words, scat- 


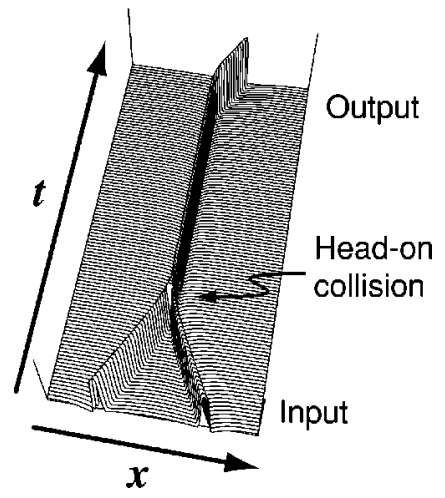

FIG. 1. Strong collision of two pulses produces just one pulse. Only the $u$ component of Eq. (3) is shown here. See Sec. IV for details of this numerics.

tors are the pivots converting one dynamic regime to another one.

It is not always true that the collision of two traveling waves produce ordered states such as pulses or spots. Figure 2 shows a transition to a complicated spatiotemporal pattern from a symmetric collision of two traveling pulses. There exists an unstable steady state (scattor) which links the traveling pulse to a spatiotemporal chaos, in fact exactly the same complicated pattern can be obtained by perturbing the scattor in an appropriate way as in Fig. 14(C), which will be discussed in Sec. III.

Various kinds of dynamic transition occur through strong collisions and the pivot called the scattor plays a traffic controller linking the input to output. Our viewpoint from scattors not only gives a clue to clarify the transient process of

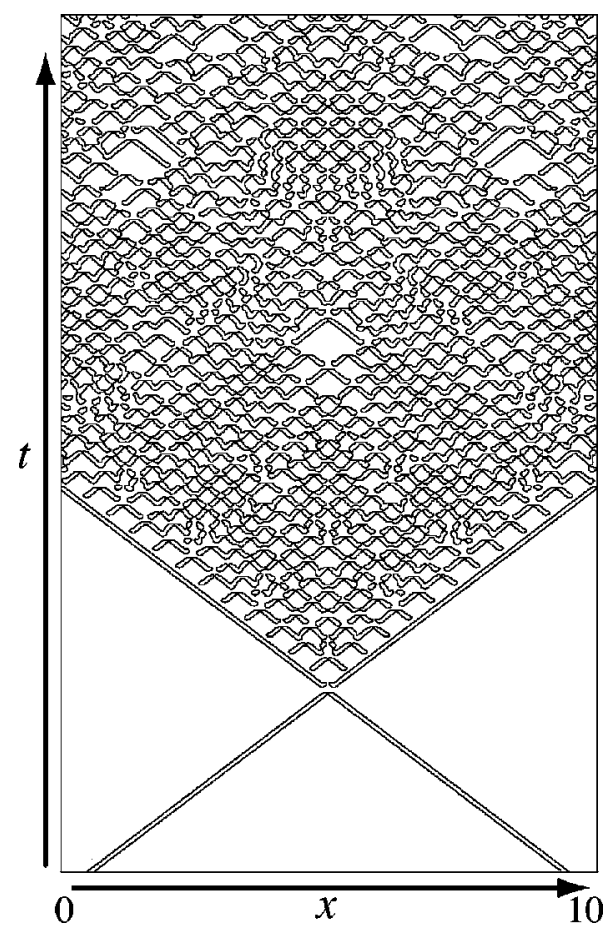

FIG. 2. Transition to chaotic regime via scattering. A symmetric collision of two pulses of Eq. (2) creates a complicated dynamics. An unstable steady state called the scattor intervenes in this transition at the colliding point. See Sec. III for details. scattering but also sheds light on the anatomy of complex dynamics like spatiotemporal chaos or chaotic itinerancy in which several different dynamic regimes coexist and the orbit is switched from one regime to another via singular events like strong collisions.

\section{SCATTOR OF CODIM 1 FOR THE COMPLEX GINZBURG-LANDAU EQUATION}

First we present a scattor of codim 1 for the complex Ginzburg-Landau equation (CGLE) with a parametric forcing term,

$$
\begin{aligned}
W_{t}= & \left(1+i c_{0}\right) W+\left(1+i c_{1}\right) W_{x x}-\left(1+i c_{2}\right)|W|^{2} W \\
& +c_{3} \bar{W},
\end{aligned}
$$

where $c_{0}, c_{1}, c_{2}$, and $c_{3}$ are real parameters. The last complex conjugate term represents an external forcing with almost double the natural frequency and $c_{2}-c_{0}$ stands for the frequency misfit. Coullet et al. ${ }^{21}$ and Sasa et al. ${ }^{22}$ have shown that Eq. (1) undergoes a supercritical drift bifurcation of domain wall and Mizuguchi and $\mathrm{Sasa}^{23}$ have found various types of instabilities by computer simulations (see also Refs. 24-26). Actually Eq. (1) is a model equation of oscillating grid pattern submitted to an oscillating external voltage in the electrohydrodynamic convection in liquid crystals. ${ }^{22,27}$ Equation (1) becomes bistable in an appropriate parameter region where there exists a pair of stable homogeneous states $W_{0}$ and $-W_{0}$ (see, for instance, Ref. 23). We employ a particular set of parameters $c_{0}=-0.15, c_{1}$ $=-0.10, c_{2}=0.10$ and vary $c_{3}$ as a bifurcation parameter in the above-mentioned bistable regime. We adopt $\Delta x \approx 0.195$, $\Delta t=10^{-3}$, the system size $=50$, and the boundary condition of Neumann type (zero flux).

When $c_{3}$ is large, the stationary front (Ising front) connecting $W_{0}$ to $-W_{0}$ (i.e., the phase of $W$ changes by $\pi$ ) is stable. Note that the magnitude of $1-|W|^{2}$ (or the modulus $|W|)$ is localized in space, so we call it a pulse rather than a front (or domain wall) in the sequel. As $c_{3}$ is decreased, a drift (Ising-Bloch) bifurcation occurs supercritically at $c_{3}$ $\approx 0.40$, which is confirmed by AUTO, ${ }^{28}$ and a stable traveling pulse emerges. The velocity of it is small near the bifurcation point and the two pulses repel each other, therefore the input-output relation is preservation, namely two incoming pulses emit two outgoing propagating pulses. As $c_{3}$ is still decreased to 0.39 , then two colliding pulses annihilate at head-on collision. Such a change of input-output relation is already reported by Ref. 12, however it remains an openquestion what mechanism actually exerts such a change. For that purpose, we carefully traced the orbital behavior near the transition point $c_{3}^{s} \approx 0.3933968$ from preservation to annihilation [see Fig. 3(A)]. It turns out that the orbit stays very close to a quasi-steady state for certain time, then it annihilates or emits two propagating pulses depending on the parameter. In fact it is numerically confirmed by using the Newton method (see, for instance, Ref. 29) that there exists a steady state $S(x)$ of codim 1, i.e., the linearized eigenvalue problem around $S(x) ; L \phi=\lambda \phi$ has only one unstable (real positive) eigenvalue $\lambda_{1}$ besides the translational zero eigenvalue [see Fig. 4(B)]. The profile of the eigenfunction asso- 

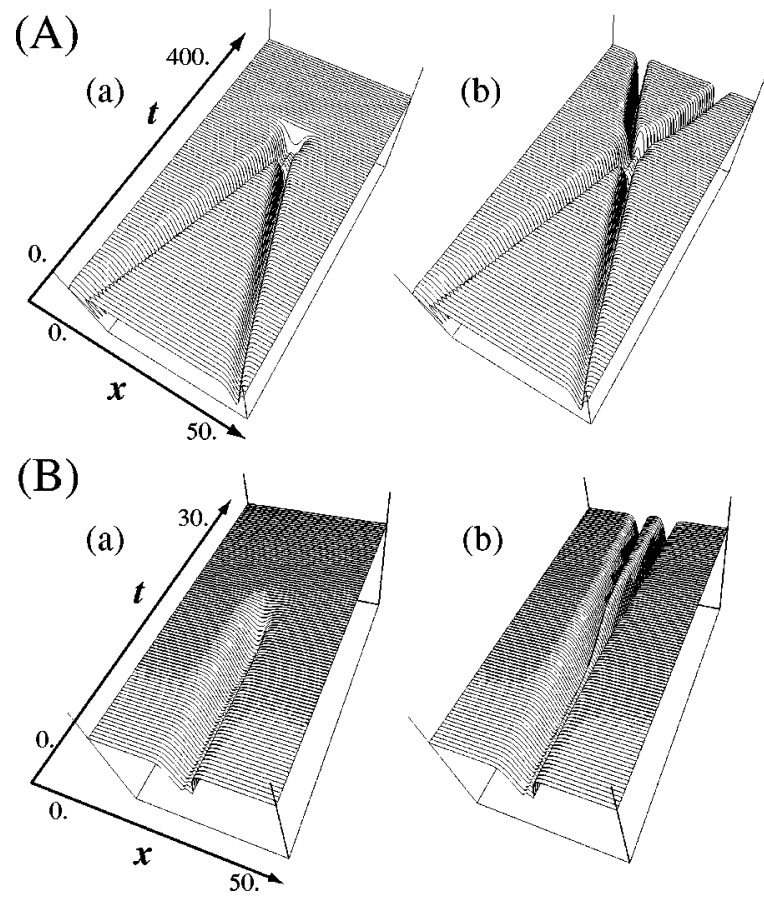

FIG. 3. Transition from reflection to annihilation. (A) The transition occurs at $c_{3}^{s} \approx 0.3933968$ from reflection (preservation) to annihilation as $c_{3}$ is decreased. [(a) Annihilation and (b) reflection, respectively.] (B) Response of the scattor $S(x)$ by adding a small perturbation in the direction of $\phi_{1}$ [see Fig. 4(B)]. (a) [respectively, (b)] Negative (respectively, positive) perturbation.

ciated with the unstable eigenvalue has a reflectional symmetry in the middle. Note that $S(x)$ depends also on $c_{3}$. Since $S(x)$ has only one unstable direction, the local dynamics around it is schematically depicted like Fig. 4(A), i.e., the stable manifold of $S(x)$ separates the phase space into two parts and the orbits are sorted out according to which side of the stable manifold it belongs. Strictly speaking we should take into account the center direction coming from the Goldstone mode, however it can be neglected, since we only con-
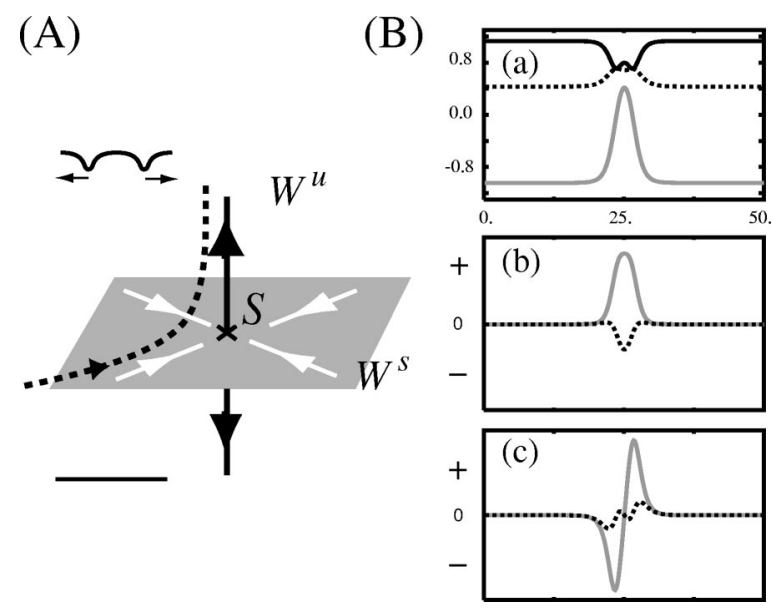

FIG. 4. Scattor for the CGLE. (A) A schematic picture of the dynamics near the scattor. (B) (a) The thick line indicates the profile of modulus of the scattor $|S(x)|$ and the gray (respectively, dotted) line shows the real (respectively, imaginary) part of it. $S(x)$ has only one (real) unstable eigenvalue $(\approx 0.4850)$ and the associated eigenfunction $\phi_{1}$ is depicted as $(b)$. The Goldstone mode is shown in (c).
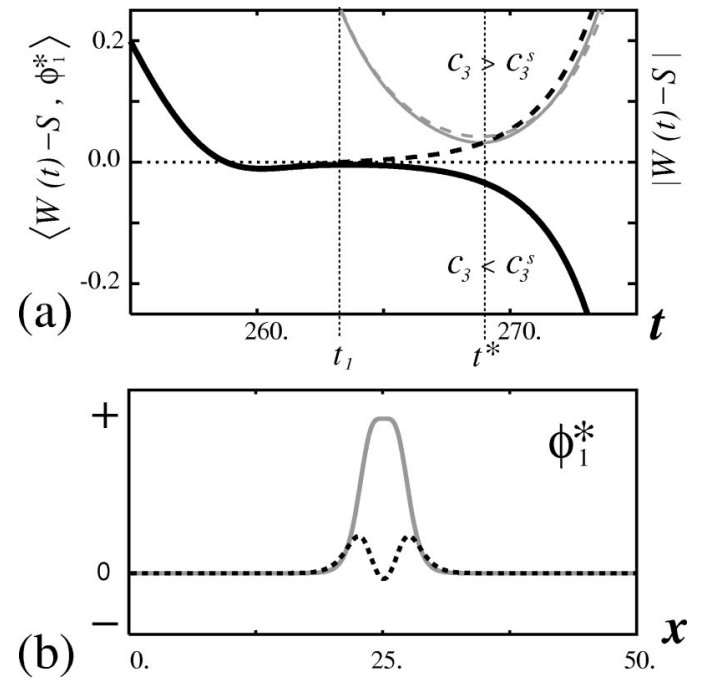

FIG. 5. Time evolution of the inner product and the adjoint eigenfunction $\phi_{1}^{*}$. (a) The dark solid (respectively, dotted) line shows the evolution of the inner product $\left\langle W(t)-S, \phi_{1}^{*}\right\rangle$ for $c_{3}=0.3933967$ (respectively, $c_{3}$ $=0.3933$ 969). When $c_{3}$ is decreased and crosses $c_{3}^{s} \approx 0.3933968$, the graph switches from positive to negative after $t=t_{1} \approx 263.3$. The gray solid (respectively, dotted) line indicates the associated evolutions of the distance between the orbit and the scattor, both of which take the minimum around $t^{*} \approx 268.8$. (b) The profile of the adjoint eigenfunction $\phi_{1}^{*}$.

sider the symmetric collisions at the center. The destinations of the unstable manifold are homogeneous state (annihilation) and counterpropagating pulses (preservation), which can be confirmed by numerics as in Fig. 3(B). In other words the transition point $c_{3}^{s}$ is characterized as the one where the orbit lies on the stable manifold, the initial condition of which is given by the two symmetric incoming true pulses colliding at the origin. Practically we employ a well-settled pulse as initial data for numerics. Here the "well-settled pulse" means that it is obtained after a long-run simulation on a large interval with periodic boundary condition. This makes sense because the concerning pulse is asymptotically stable. Quantitatively the time evolution of the inner product $\left\langle W(t)-S, \phi_{1}^{*}\right\rangle$ may give useful information to predict the fate of the orbit, where $W(t)$ is a solution to Eq. (1) and $\phi_{1}^{*}$ is the adjoint eigenfunction of $\lambda_{1}$. In fact we see clearly as in Fig. 5 that the inner product changes its sign after collision from positive to negative as $c_{3}$ is decreased via $c_{3}^{s}$. This is a natural consequence in view of the separating behavior in Fig. 4(A). It is convenient to introduce $t^{*}=t^{*}\left(c_{3}\right)$ defined by the time when the distance $\left|W\left(t ; c_{3}\right)-S\left(x ; c_{3}\right)\right|$ attains the minimum during the collision process. Here the distance is measured by the square integral of the function, i.e., $L^{2}$ norm. The associated solid (respectively, dotted) gray line in Fig. 5(a) shows how the above-mentioned distance evolves and the location of the minimum point $t^{*} \approx 268.8$. The transition point $c_{3}^{s}$ is characterized as $t^{*}\left(c_{3}^{s}\right)=+\infty$, since the orbit $W(t)$ lies on the stable manifold of $S(x)$ at $c_{3}=c_{3}^{s}$. Moreover if one could find two points with opposite signs of $\left\langle W\left(t^{*}\left(c_{3}\right) ; c_{3}\right)-S\left(x ; c_{3}\right), \phi_{1}^{*}\right\rangle$ as a function of $c_{3}$, any value in between gives a good approximation of $c_{3}^{s}$, and the sign of it offers a simple criterion for the output, namely, if it is positive (respectively, negative), it implies two-pulse emis- 


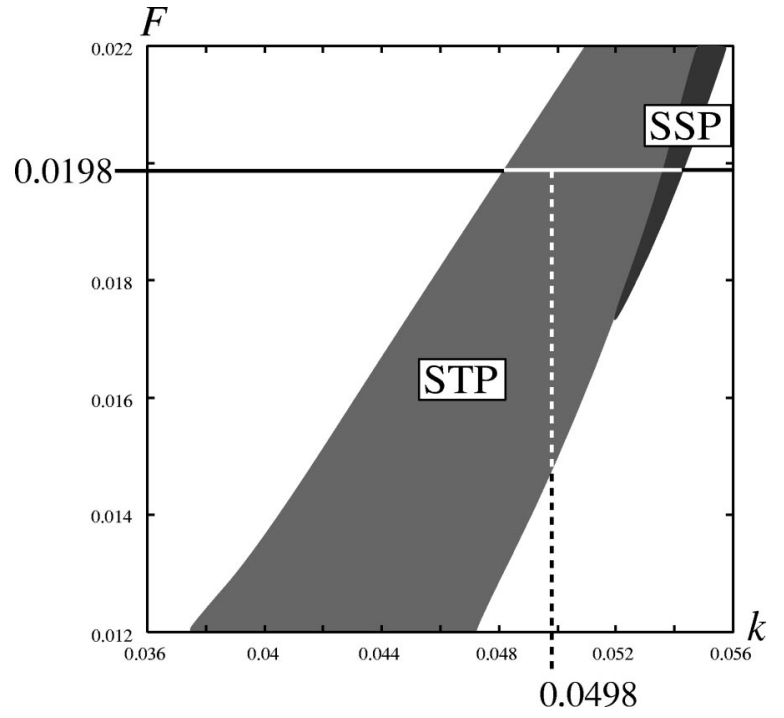

FIG. 6. Existence region of stable traveling pulse for the Gray-Scott model. Stable one-dimensional traveling (standing) wave is observed in STP (SSP). When $F$ is fixed to be 0.0198 , the transition occurs from annihilation to repulsion (preservation) at $k=0.0498$. See the text for details.

sion (respectively, annihilation). We call $S(x)$ a scattor for Eq. (1), which controls the traffic of flows and is responsible for the transition of input-output relation. We can classify the possible outputs after collisions and detect the location of the transition points through $S(x)$. In the following sections we shall show that such a scattor is ubiquitous in a variety of scattering process in dissipative systems. In Ref. 30 we used the terminology "separator" instead of scattor, however scattor may be appropriate especially for higher codimension case.

\section{SCATTOR OF HIGHER CODIMENSION FOR THE GRAY-SCOTT MODEL}

A transition from repulsion to annihilation is observed for the CGLE in Sec. II. Although its modulus $|W|$ is localized in space, it is an interaction between the two fronts. Such a transition also occurs for real localized traveling pulses and we employ the following Gray-Scott (GS) model

(2) as a representative one (see Ref. 31):

$$
\begin{aligned}
& u_{t}=D_{u} \Delta u-u v^{2}+F(1-u), \\
& v_{t}=D_{v} \Delta v+u v^{2}-(F+k) v,
\end{aligned}
$$

where $F>0$ and $k>0$ are parameters related to inflow and removal rate of chemical species. It is known that Eq. (2) has a stable traveling pulse in an appropriate parameter region (Fig. 6). In what follows, we consider the case in which $F$ is fixed to be either 0.0198 or 0.0194 and $k$ varies as a bifurcation parameter. For all simulations for the GS model to follow, we used the explicit scheme with $\Delta t=10^{-2}, \Delta x$ $=0.005, D_{u}=5.0 \times 10^{-5}$, and $D_{v}=2.5 \times 10^{-5}$. Also we only consider the symmetric collision, namely the initial two pulses are perfectly symmetric except their propagating directions. In other words, it is equivalent to hitting a wall under Neumann boundary condition. As initial data we employ a well-settled symmetric pair of pulses introduced in
Sec. II. For the scattering of asymmetric initial condition, we refer to Ref. 30. When $k$ is increased and exceeds $k_{c}$ $\approx 0.0497859$, the input-output relation changes from annihilation (A) to repulsion (B) as in Fig. 7. A remarkable thing is that there appears a quasi-steady state of twin-horn shape right after collision and the orbit approaches it, stays there for certain time, then annihilates or emits two pulses. In fact there exists a real steady state of twin-horn shape, which is numerically confirmed by the Newton method. A linearized eigenvalue problem, $L \phi=\lambda \phi$, where $L$ is the linearized operator of the right-hand side of the system (2) around the twin-horn steady state has three unstable eigenvalues $\lambda_{1}$ $=0.06389>\lambda_{2}=0.06378>\lambda_{3}=0.00233$ besides the zero eigenvalue $\lambda_{4}$ coming from the translation invariance [see Fig. 7(C)]. Note that the first two eigenvalues are much larger than the third one, hence the dynamics is basically controlled by $\lambda_{1}$ and $\lambda_{2}$. The associated eigenfunctions are denoted by $\phi_{i}(i=1, \ldots, 4)$. The twin-horn scattor plays a role as a traffic controller at collision. In fact, for symmetric head-on collision, the second eigenfunction $\phi_{2}$ plays an important role to determine the fate of the orbit, namely, adding its small constant-multiple perturbation to the twin-horn pattern, then the resulting behavior is either annihilation or emission of two pulses depending on its sign of constant [Figs. 8(c) and 8(d)]. In other words the output can be classified by looking at the response of the scattor along the unstable manifold. It should be noted that the scattor can be obtained by continuation of a stable standing pulse as in Fig. 9, which not only shows that the scattor $S(k)$ depends smoothly on $k$ in a wider interval of $k$, but also it is connected to the observable patterns.

Recalling that only symmetric collisions are considered and $\lambda_{2} \gg \lambda_{3}$, the inner product $\left\langle U(t, x)-S(k), \phi_{2}^{*}\right\rangle$ similar to the one in the CGLE case serves to detect the transition point $k_{c}$ and predicts the orbital behavior near $S(k)$ despite codim $S(k)>1$, where $U(t, x)$ denotes the solution to Eq. (2), and $\phi_{2}^{*}$ the adjoint eigenfunction associated with $\lambda_{2}$. The time evolutions of the inner products for $k=0.0497$ and 0.0499 are depicted in Fig. 10(a), which gives us a criterion that $k_{c}$ is approximated well by the point where the sign of $\left\langle U\left(t^{*}, k\right)-S(k), \phi_{2}^{*}\right\rangle$ changes from negative (annihilation) to positive (repulsion) as $k$ is increased. Here $t^{*}$ is defined similarly as in Sec. II. Note that, unlike the CGLE case, $t^{*}$ for the GS case generically remains finite around $k_{c}$ owing to the fact that codim $S(k)>1$. This criterion has a limitation due to its linearity and codim $S(k)>1$. Nevertheless it gives a practical criterion for detecting the transition point $k_{c}$.

A complete different type of transition exists for smaller $k$ values. In fact the annihilation region in Fig. 11 is bounded by two curves $\Gamma_{1}$ and $\Gamma_{2}$. The line $\Gamma_{1}$ in Fig. 11 shows a transition of input-output relation from repulsion to annihilation as we discussed earlier. The transition from annihilation to spatiotemporal chaos occurs when the parameters cross the line $\Gamma_{2}$ and enter into the region $\mathrm{C}^{\prime}$. For a fixed $F=0.0194$, the scattering processes on both sides of $\Gamma_{2}$ are depicted in Fig. 12. A single pulse is still asymptotically stable in the region $\mathrm{C}^{\prime}$, however when it collides with another pulse, two counterpropagating pulses are emitted, but those have splitting instabilities [Fig. 12(a)] and produce a 


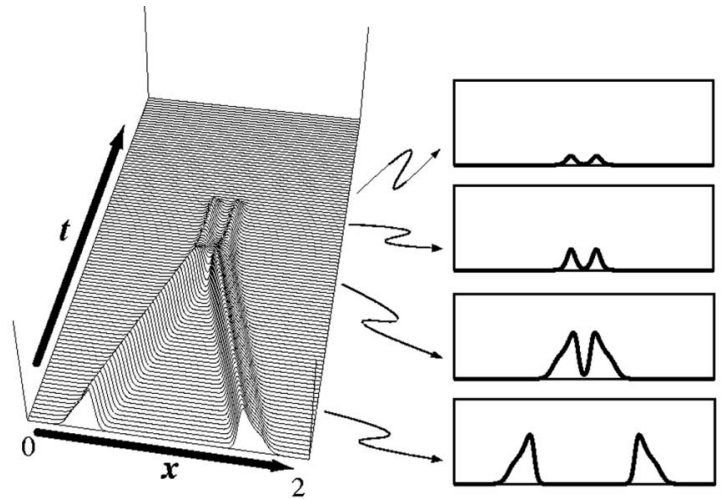

(A)

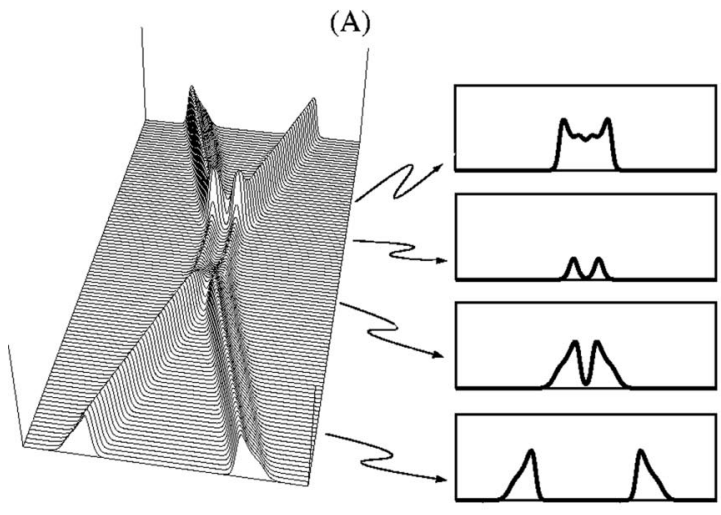

(B)

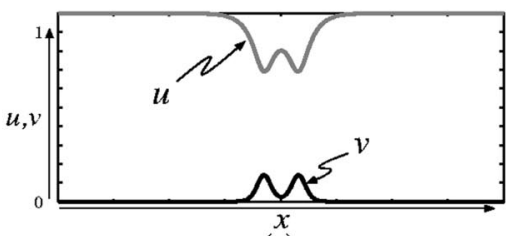

(a)

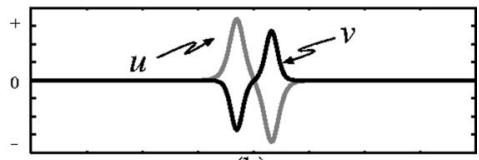

(b)

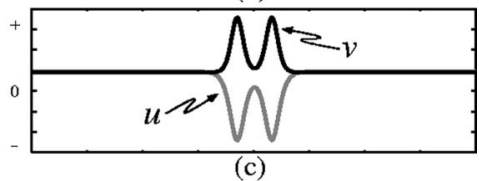

(c)

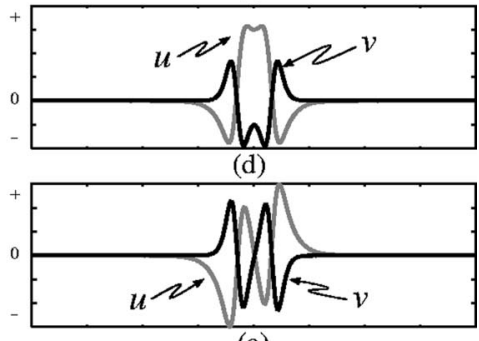

(C)
FIG. 7. Symmetric collisions for $F$ $=0.0198$. (A) Annihilation occurs at $(k, F)=(0.0497859,0.0198)$. As $k$ is slightly increased to 0.0497860 , transition from annihilation to repulsion occurs. Note that just before the occurrence of annihilation or creation of counterpropagating pulses, both orbits in (A) and (B) stay very close to the scattor depicted in (C) (a). Only the $v$ component is shown in (A) and (B). (C) (a) The profile of the unstable steady state of codim 3 (scattor). Three unstable eigenfunctions $\phi_{1}, \phi_{2}, \phi_{3}$ are depicted as (b) -(d), and (e) corresponds to the Goldstone mode. The associated eigenvalues are $\lambda_{1}=0.06389>\lambda_{2}$ $=0.06378>\lambda_{3}=0.00233$. The first two eigenvalues are much larger than the third one. The solid (gray) line indicates the $v(u)$ component. complicated spatiotemporal pattern after a long run as in Fig. 13(b) (recall that hitting the boundary with Neumann condition is equivalent to a symmetric collision). On the other hand, if the parameters are slightly shifted to the right-hand side of $\Gamma_{2}$, then we have an annihilation like Fig. 12(b). In

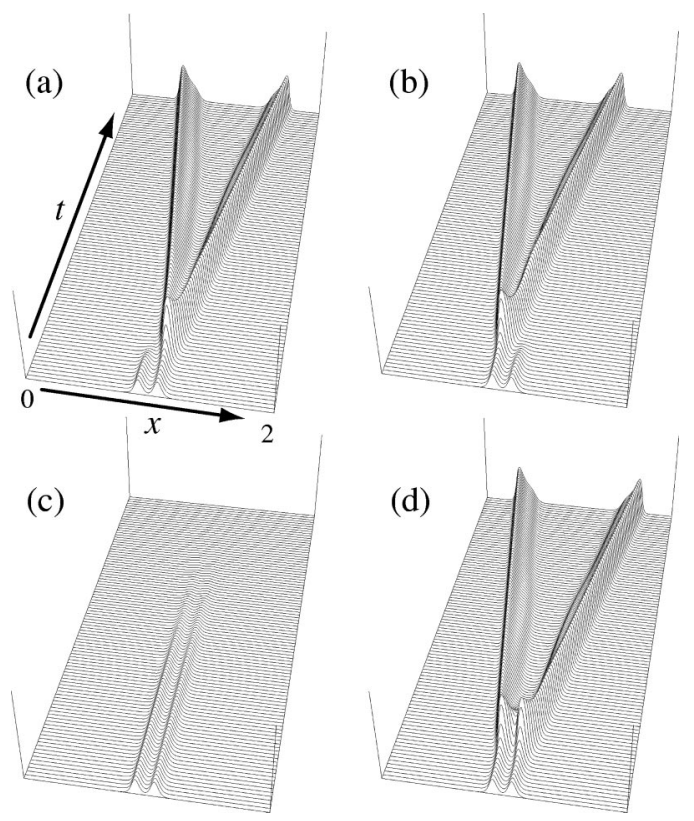

FIG. 8. Outputs from the scattor for $F=0.0198$. (a) [respectively, (b)]: A small positive (respectively, negative) perturbation of $\phi_{1}$ is added to the twin-horn scattor. (c) [respectively, (d)]: A small negative (respectively, positive) perturbation of $\phi_{2}$ is added to the scattor. The output of annihilation (respectively, two-pulse emission) is consistent with that of Fig. 7. fact, two different dynamic regimes coexist in the region $\mathrm{C}^{\prime}$ : one is stable traveling pulses and the other is self-splitting pulses which eventually create a complicated pattern. Although we do not discuss details of this complicated pattern here, a remarkable thing is that there is a scattor which converts an ordered state (traveling pulse) to a spatiotemporal

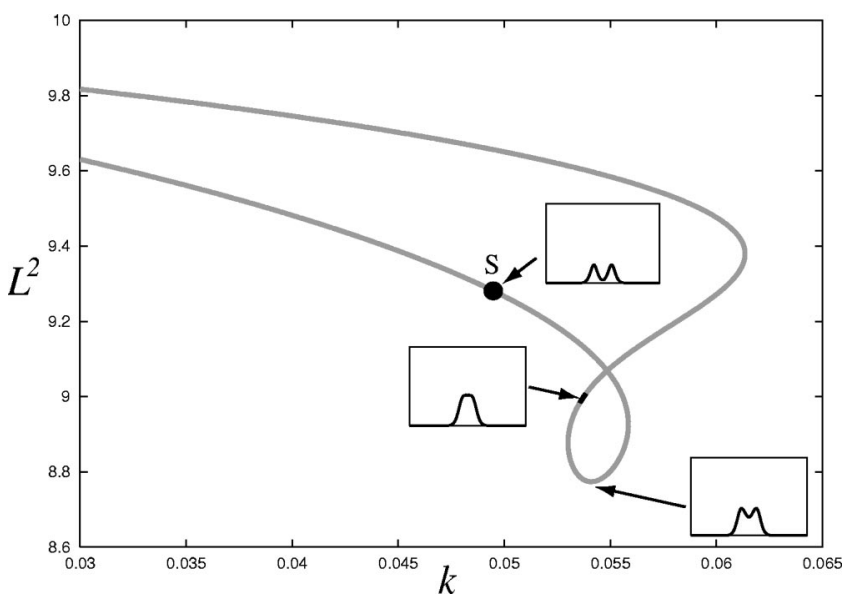

FIG. 9. Global bifurcation diagram for the twin-horn scattor of the GS model. The bifurcation parameter (horizontal axis) is $k$ with $F$ being fixed as 0.0198 . The ordinate $L^{2}$ stands for the integral norm of square of $u$ and $v$. The solid (respectively, gray) line indicates the stable (respectively, unstable) part. The twin-horn scattor of Fig. 7 is designated by $S$. Note that it is connected to a stable standing pulse. The branch looks intersecting with itself, however this is an apparent intersection due to projection. Here we used AUTO (Ref. 28) to compute the branch globally. 


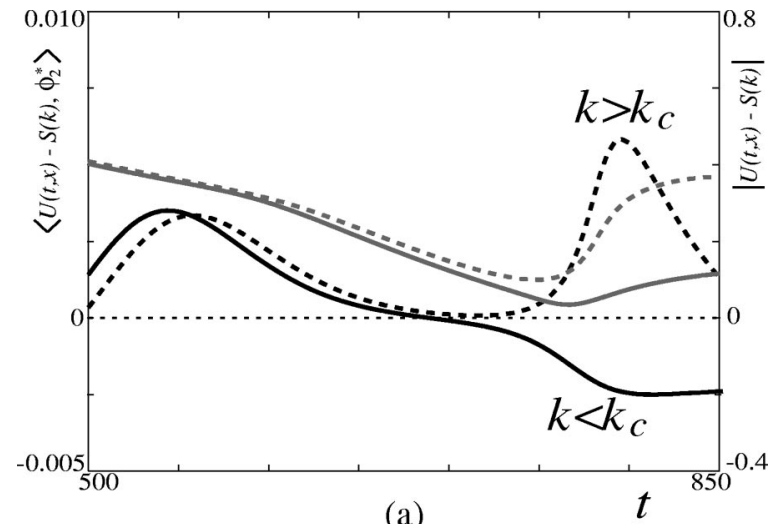

(a)

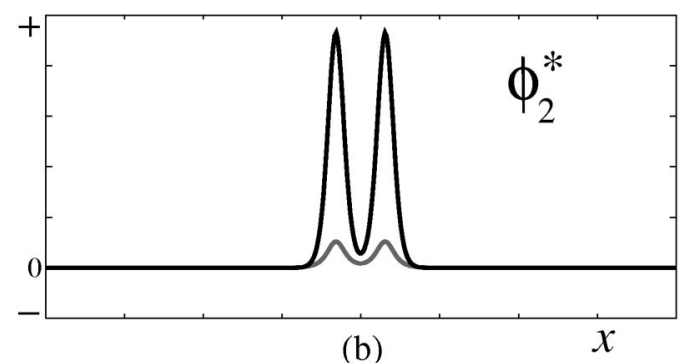

(b)

FIG. 10. Time evolution of the inner product for the GS model and the adjoint eigenfunction $\phi_{2}^{*}$. (a) The dark solid (respectively, dotted) line shows the evolution of the inner product $\left\langle U(t, x)-S(k), \phi_{2}^{*}\right\rangle$ for $k$ $=0.0497$ (respectively, $k=0.0499$ ). When $k$ is increased and crosses $k_{c}$ $\approx 0.04978$, the graph switches from negative to positive. The gray solid (respectively, dotted) line indicates the associated evolutions of the distance between the orbit and the scattor for $k=0.0497$ (respectively, $k=0.0499$ ). (b) The profile of the adjoint eigenfunction $\phi_{2}^{*}$.

chaos. The scattor sitting on the line $\Gamma_{2}$ again looks like a twin-horn shape as in Fig. 14(D)(a) of codim 3 and when we perturb it in the most unstable direction of symmetric shape [Fig. 14(D)(c)], it produces either annihilation or spatiotemporal chaos as in Figs. 14(A)-14(C). Note that scattors form a one-parameter family of solutions like Fig. 10. What happens around $\Gamma_{1}$ and $\Gamma_{2}$ is that the orbit starting from a pair of symmetric pulses crosses the augmented stable manifold of the scattor by $\phi_{3}$, and hence its destination is changed leading to the transition of input-output relation. The orbit therefore comes closer to the scattor near those transition points. In terms of inner product this is equivalent to the change of sign of $\left\langle U\left(t^{*}, k\right)-S(k), \phi_{2}^{*}\right\rangle$ as a function of $k$. In this sense the scattor is a kind of hinge connecting two different dynamic regimes through scattering. Finally if parameters belong to the region $\mathrm{C}$ in Fig. 11, there are no stable traveling pulses and the initial pulse immediately bears daughter ones and eventually produces a complicated pattern as in Fig. 13(a).

\section{BIFURCATION OF SCATTORS AND MULTIPLE SCATTOR}

Such a scattor may exist in a wider class of dissipative systems in which traveling waves are observed. We illustrate this by using a three-component reaction diffusion system (3), which was proposed as a qualitative model of gas discharge system ${ }^{32}$ and displays a variety of dynamic patterns including particle-like objects called dissipative solitons: ${ }^{2,33}$
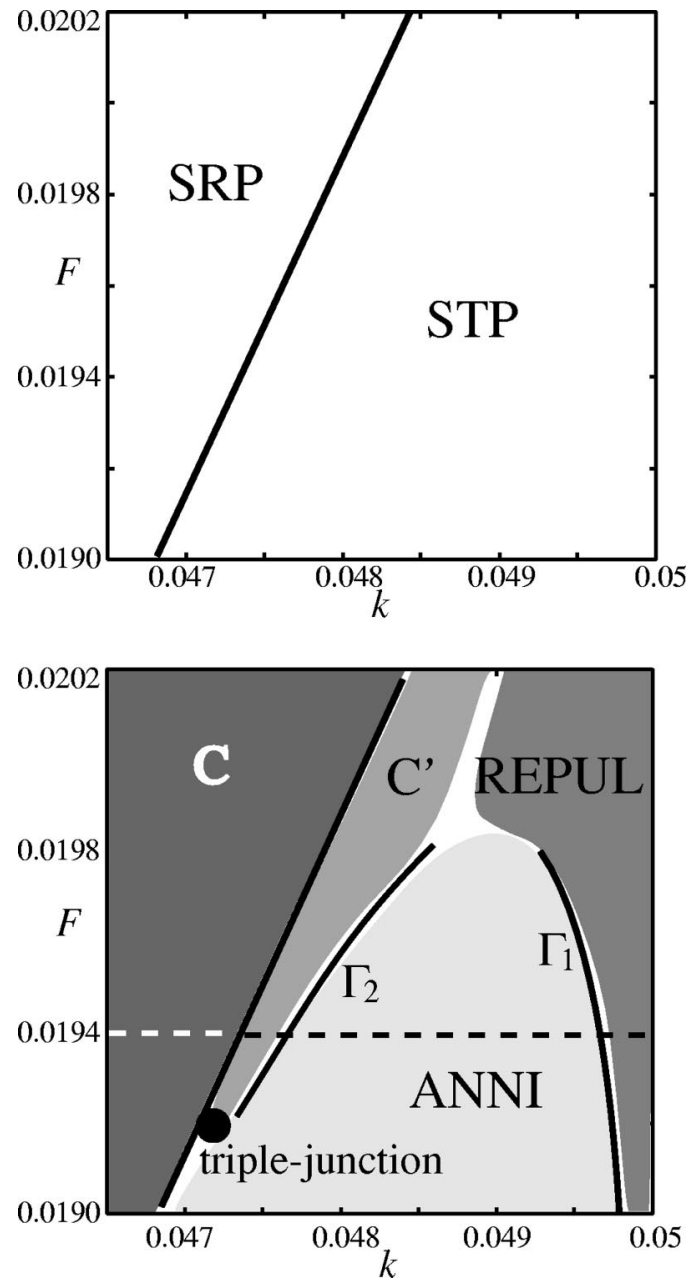

FIG. 11. Classification of the output after symmetric collision. Existence region of self-replicating pattern (SRP) and stable traveling pattern (STP) (upper panel). Phase diagram of the outcome after symmetric collision (lower panel). C: spatiotemporal chaos, $\mathrm{C}^{\prime}$ : spatiotemporal chaos after collision, REPUL: repulsion, and ANNI: annihilation. There exists a triplejunction point where regions $\mathrm{C}, \mathrm{C}^{\prime}$, and $\mathrm{ANNI}$ meet.

$$
\begin{aligned}
& u_{t}=D_{u} \Delta u+f(u)-\kappa_{3} v-\kappa_{4} w+\kappa_{1}, \\
& \tau v_{t}=D_{v} \Delta v+u-v, \\
& \theta w_{t}=D_{w} \Delta w+u-w,
\end{aligned}
$$

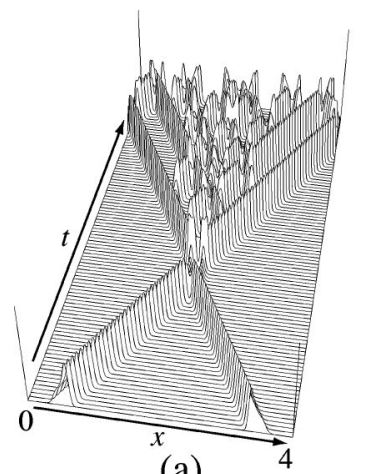

(a)

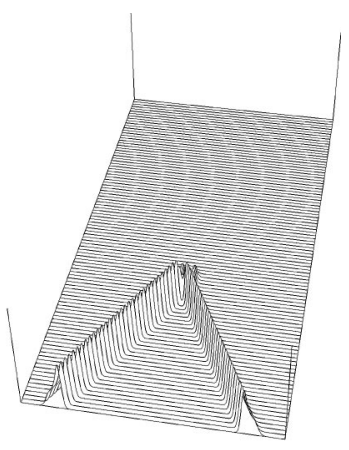

(b)
FIG. 12. Transition from chaotic regime to annihilation. Input-output relation changes when the parameters cross $\Gamma_{2}$. (a) $F=0.0194, k$ $=0.0476430$ ( $\mathrm{C}^{\prime}$ region). (b) $F=0.0194, k=0.0476431$ (ANNI region). Only the $v$ component is shown here. 


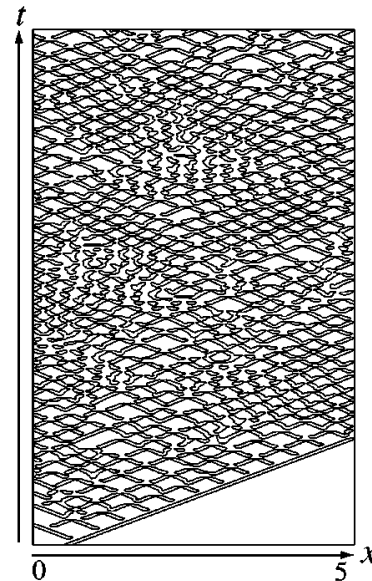

(a)

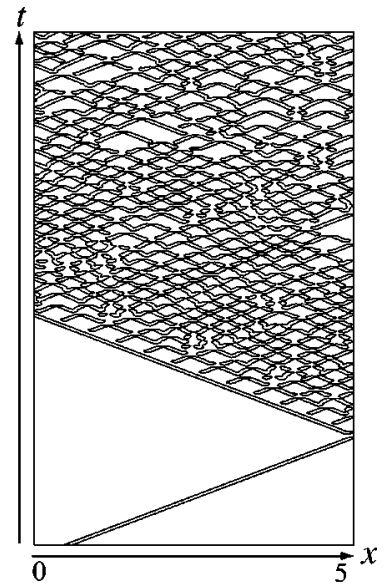

(b)
FIG. 13. Plots of the contour lines $v=0.2$ of typical orbits in $\mathrm{C}$ and $\mathrm{C}^{\prime}$ $F=0.0194$ for both computations. (a) In region $C(k=0.04735)$ the pulse immediately starts to split and shows a complicated behavior. (b) In region $\mathrm{C}^{\prime}(k=0.0475)$ the stable pulse exists, but it is switched to a regime of spatiotemporal chaos after the symmetric collision.

where we set $f(u)=2 u-u^{3}$. We consider Eq. (3) with $\left(D_{u}, D_{v}, D_{w}\right)=\left(5.0 \times 10^{-6}, 5.0 \times 10^{-5}, 1.0 \times 10^{-2}\right)$ and $\tau$ being a bifurcation parameter. The other parameters are set to be $\kappa_{1}=-7.0, \kappa_{3}=1.0, \kappa_{4}=8.5$, and $\theta=1.0$. In order to integrate Eq. (3), we used a semi-implicit scheme with $\Delta x$ $=2^{-10}$ and $\Delta t=10^{-2}$ and the system size is either 0.5 or 1.0 subject to Neumann boundary conditions. This type of threecomponent reaction diffusion system is an appropriate setting for the study of scattering of particle-like solutions in higher dimensional space. ${ }^{33}$ Here we focus on the symmetric collisions in one-dimensional space. The input-output diagram is depicted as in Fig. 15(a) as well as the bifurcation diagram for the standing pulse as $\tau$ varies. The initial data are taken to be well-settled pulses as in the GS case. Traveling pulses bifurcate supercritically at $\tau \approx 9.7\left(\equiv \tau^{d}\right)$ and they are repulsive near the bifurcation point, in fact they scatter like Fig. 15(b) (left). The input-output relation is however switched from two-pulse-emission (repulsion) to one-pulseemission at $\tau^{s} \approx 16.1328079$. Scattors are again the key to understanding this transition, in fact there are two scattors involved during the scattering process; one is the twin-horn pattern of codim 3 [Fig. 16(d)], similar to the GS case, and the other is the fusion solution (standing pulse) of codim 1 [Fig. 16(b)]. For $\tau$ being slightly smaller than $\tau^{s}$, the orbit approaches the twin-horn pattern but eventually leaves and repels each other. On the other hand when $\tau$ is slightly larger than $\tau^{s}$, the orbit first approaches the twin-horn pattern, then its middle part rises and becomes very close to the fusion pattern [see the magnified picture of Fig. 16(a)]. Recalling that the fusion solution has a drift instability for $\tau>\tau^{d}$, it starts to move either to the left or right after some waiting time [Fig. 16(a)]. Note that it is not predictable in which direction the pulse eventually moves, since it comes from tiny round-off errors. It is quite remarkable that the orbit passes by two different scattors successively; twin-horn type and fusion type. Although the twin-horn scattor has three unstable eigenvalues, the first one is much larger than others
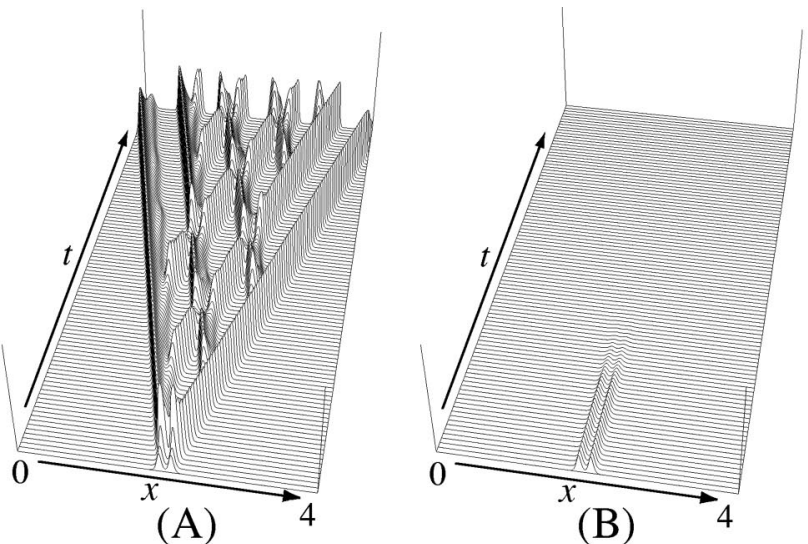

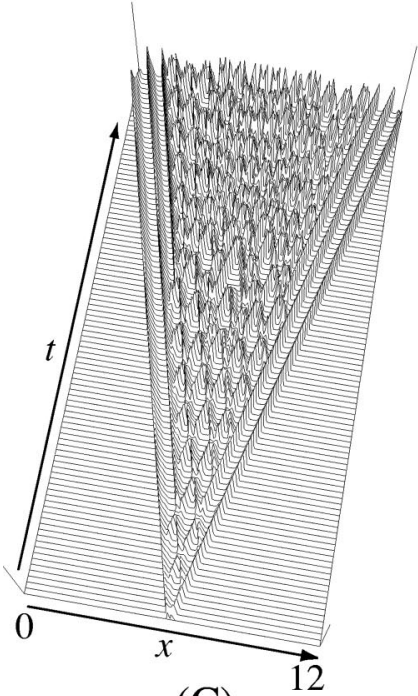

(C)
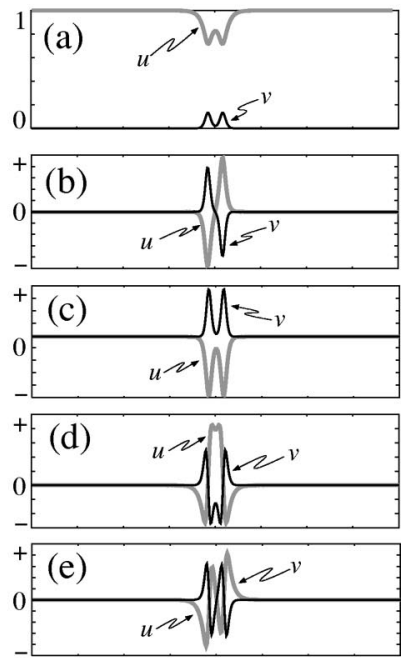

(D)
FIG. 14. Response of the scattor $(F=0.0194)$. There exists a scattor of twin-horn shape like (D) (a) and adding a small perturbation proportional to the second eigenfunction (D) (c), then, depending on its sign, the output is either a spatiotemporal chaos (negative) (A) or annihilation (positive) (B). (C) is a longer time simulation of (A) on a larger system size. (D) A profile of the scattor (a) and the associated eigenfunctions (b)-(e) similar to Fig. $7(\mathrm{C})$.

$\left(\lambda_{1}=0.9069>\lambda_{2}=0.1297>\lambda_{3}=0.0138\right)$ and hence the dynamics is basically controlled by it. In view of the eigenform $\Phi_{1}$ associated with $\lambda_{1}$ [Fig. 16(e)], it is symmetric and has a high peak in the middle, which drives a motion from twinhorn to fusion. The transition point $\tau^{s}$ can be characterized in a similar way as in the GS case, namely $\tau^{s}$ is given by the zero point of the inner product $\left\langle U\left(t^{*}(\tau), x\right)-S(\tau), \Phi_{1}^{*}\right\rangle$ where $U\left(t^{*}, x\right)$ denotes the solution profile to Eq. (3) at $t$ $=t^{*}, S(\tau)$ the scattor of the twin-horn shape, $\Phi_{1}^{*}$ the adjoint eigenfunction associated with $\Phi_{1}$, and $t^{*}$ is defined similarly as before. Moreover this gives a criterion that if the inner product is negative (respectively, positive), it emits two pulses (respectively, one pulse) with the same caveat as in the GS case. The above $\tau^{s}$ is theoretically defined when the initial data for $U\left(t^{*}, x\right)$ is taken as a symmetric pair of true pulses on the whole line. Numerically, as in the GS case, $\tau^{s}$ is well approximated by using well-settled pulses as initial data. Still $\tau$ is increased, the input-output relation remains the same, however the dynamics during the scattering process becomes oscillatory as in the right figure of Fig. 15(b). 

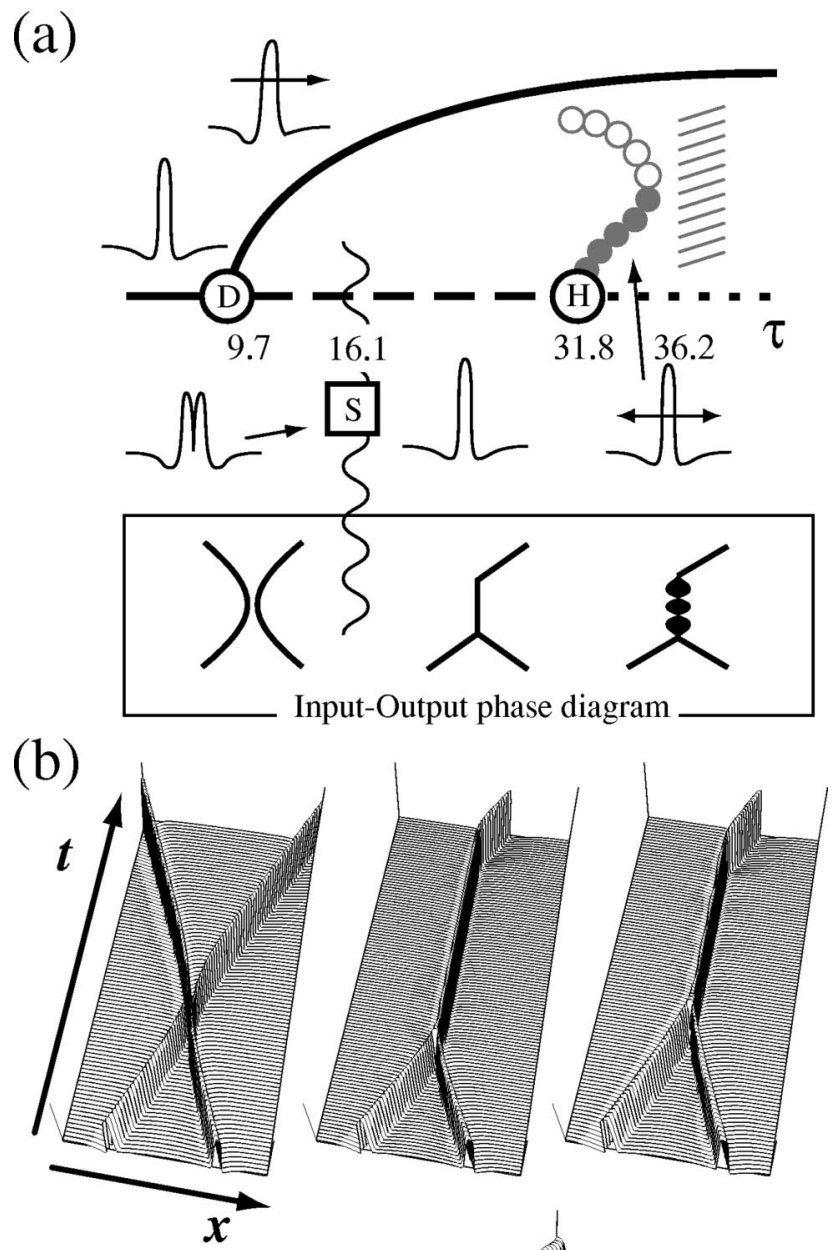

(c)

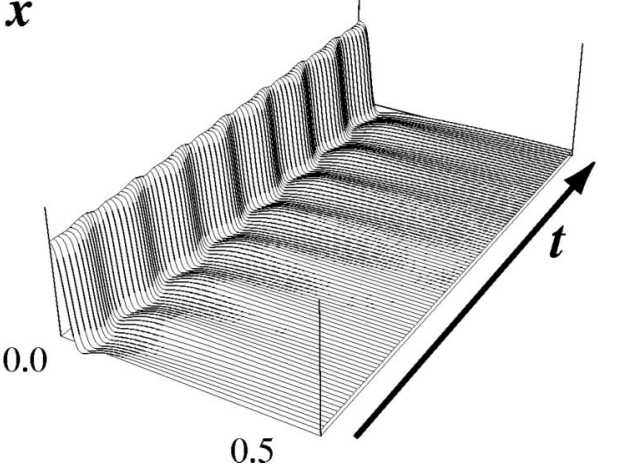

FIG. 15. (a) Schematic phase diagram for the gas-discharge system: D (respectively, H) denotes the drift (respectively, Hopf) bifurcation of the standing pulse as $\tau$ is increased. Stable traveling pulses emanate from D and time-periodic solutions of codim 1 bifurcate from $\mathrm{H}$ as is shown in (c). The periodic branch has a saddle-node point around $\tau \approx 36.2$. $\mathrm{S}(\approx 16.1)$ indicates the transition point of input-output relation from two-pulse-emission to one-pulse-emission as $\tau$ is increased. The lower column shows a schematic diagram of input-output relations in which black disk indicates an oscillation during the scattering process. (b) Bird's-eye views of inputoutput for several $\tau$ values (left: $\tau=15.0$, center: $\tau=20.0$, right: $\tau=35.0$ ). The initial functions are taken to be a snapshot of well-settled traveling pulse. The original simulations were done for the system size being equal to 1 , however the central parts of them are displayed here. (c) Oscillatory scattor for $\tau=35.0$ which bifurcates from $\mathrm{H}$ and has a drift instability, however it is observable on a half-space with zero-flux boundary conditions by suppressing the drift instability. The system size is 0.5 .

This is because the fusion pattern undergoes a Hopf bifurcation at $\tau \approx 31.8$ and the steady fusion scattor is replaced by the time-periodic one [see the Hopf branch of the gray color in Fig. 15(a)]. The new time-periodic scattor has only a drift
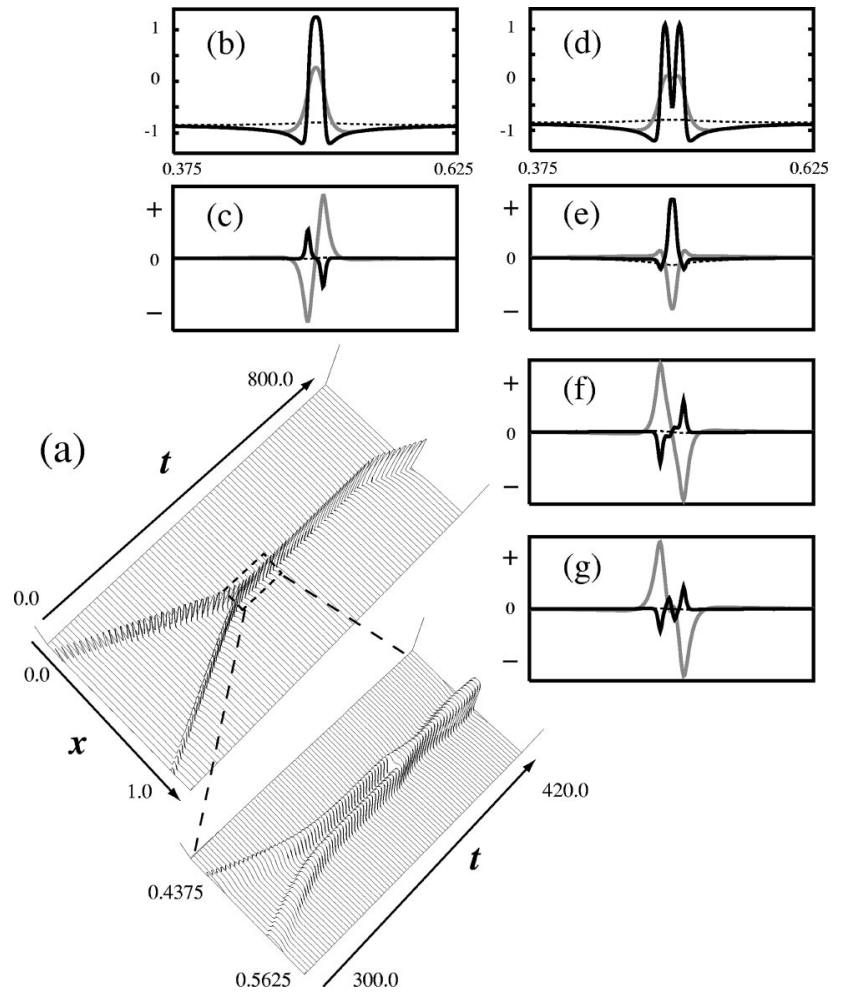

FIG. 16. Scattors of the fusion and twin-horn type: (a) Transition from repulsion to one-pulse emission occurs when $\tau \approx \tau^{s}$. When $\tau$ is slightly larger than $\tau^{s}$, the orbit traverses two different types of scattors successively. Only the $u$ component is shown here. (b) The profile of the fusion pattern of codim 1 and the unstable eigenfunction are depicted in (c). (d) The profile of twin-horn scattor of codim 3. Three unstable eigenvalues $\lambda_{1}=0.9069>\lambda_{2}$ $=0.1297>\lambda_{3}=0.0138$ and the associated eigenfunctions $\Phi_{1}, \Phi_{2}$, and $\Phi_{3}$ are shown as (e)-(g). We omit the Goldstone mode here. The solid, gray, and broken lines indicate $u, v$, and $w$ components, respectively. The other parameters are the same as in Fig. 15 and the system size is 1.0.

instability, therefore suppose a collision occurs in a perfectly symmetric way, or equivalently, the pulse collides with a boundary with Neumann boundary condition, which suppresses the drift instability, then it stays there as a stable time-periodic solution like Fig. 15(c). On the other hand, on an extended domain, a tiny fluctuation causes a drift bifurcation and emits a single pulse as in the right figure of Fig. 15(b).

\section{SCATTORS IN TWO-DIMENSIONAL SPACE}

For the two-dimensional (2D) case it is not at all trivial to have a stable traveling spot, in fact two-component reaction diffusion systems in general do not support such moving patterns. ${ }^{33}$ The third species somehow plays an important role to keep the shape of the spot firmly. The threecomponent system (3) turns out to serve as a representative model for our purpose. We have a variety of scattering processes including oblique collisions. Head-on collision may not be generic in higher dimensional space, however qualitative properties such as the number of particles or topology of localized patterns may be changed only at strong collision. It should be remarked that the anisotropy coming from the crystalline structure of material (see Ref. 5) in the CO oxi- 


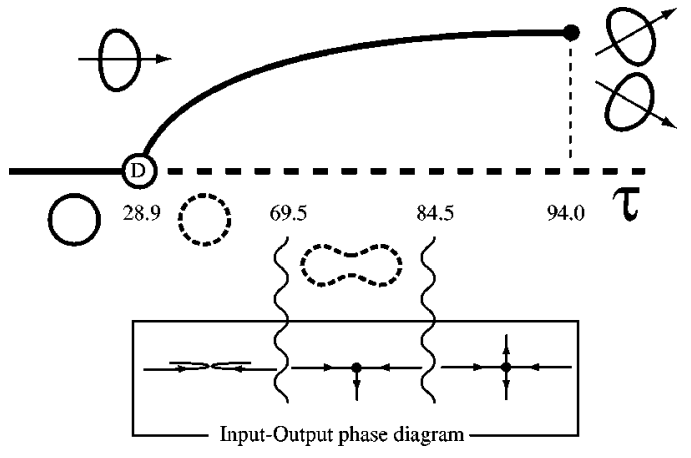

FIG. 17. Schematic phase diagram for the two-dimensional case: $\mathrm{D}$ denotes the drift bifurcation of the standing spot as $\tau$ is increased. When $\tau$ is larger than 94.0, the spot starts to split and there are no stable traveling spots.

dation reaction $\mathrm{Pt}(110)$ offers a field where traveling spots have a preference of propagating direction so that they have much more chance to have head-on collisions. We employ the following parameter values for the $2 \mathrm{D}$ case of Eq. (3): $\left(D_{u}, D_{v}, D_{w}\right)=\left(0.9 \times 10^{-4}, 1.0 \times 10^{-3}, 1.0 \times 10^{-2}\right)$ and $\kappa_{1}$ $=-7, \kappa_{3}=1, \kappa_{4}=8.5$, and $\theta=1$. See captions for other numerical data. We set $\Delta x=2^{-7}$ and $\Delta t=10^{-2}$ and the fivepoint difference approximation of the Laplacian is used. The system size is $2.0 \times 1.0$. Here we employ $\tau$ as a control parameter, although there may be other equivalent options to have similar transitions. When $\tau$ is small, there exist stable standing spots and they interact in a repulsive way. As $\tau$ is increased, a standing spot undergoes a drift bifurcation at $\tau_{d} \approx 28.9$ and a traveling spot emerges supercritically (Fig. 17). The resulting traveling spots still interact in a repulsive way near the bifurcation point and when they make a head-on collision, the input-output looks like a bouncing ball [Fig. 18(A)]. As $\tau$ is increased, the first qualitative change of the input-output relation occurs around $\tau_{c}$ $\approx 69.54853$, i.e., repulsion is switched to emitting one-spot [Figs. 18(A) and 18(B)]. Taking a closer look at the behaviors of the moving spots in the neighborhood of $\tau_{c}$, there are two kinds of scattors sitting there and the orbital behaviors are sort out by them. Note that scattors in general exist in a wider region of the parameter space, however they are highly unstable and become visible only when orbits come close to them at transition points. When $\tau$ is slightly smaller than $\tau_{c}$, the two spots come close and form a shape like a gourd for a while [Fig. 18(A) $t=200$ ], then repel each other. On the other hand when $\tau$ is slightly larger than it, the two spots first take a gourd-like shape after collision [Fig. 18(B) $t=200$ ], then the central part of the gourd rises and becomes a fused spot [Fig. 18(B) $t=300$ ] and it keeps its shape for certain time, and finally the fused spot starts to move in one direction [Fig. 18(B) $t=600$ ]. It is numerically confirmed by the Newton method that both gourd shape pattern and fused spot exist as steady states of Eq. (3). They are unstable of codim 5 and codim 2 (Figs. 19 and 20) besides two Goldstone modes and play the role of scattors.

In fact, this is a $2 \mathrm{D}$ version of the two-step scattering process we saw for the one-dimensional case in Sec. IV. The transient process around $\tau_{c}$ can be described and classified just by looking at the outputs from those scattors. For in-

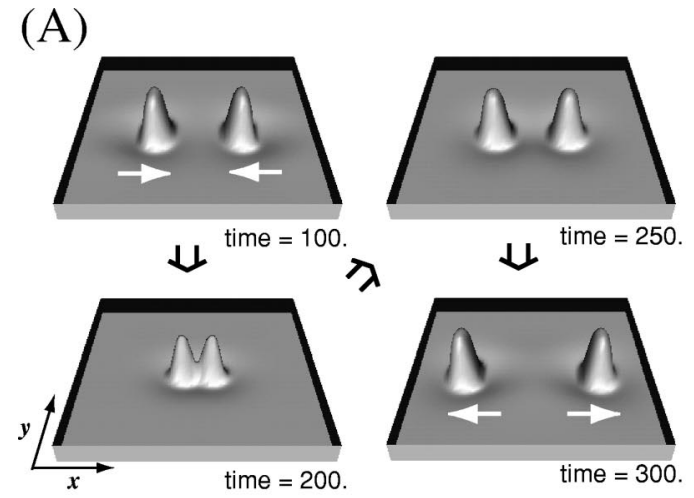

(B)

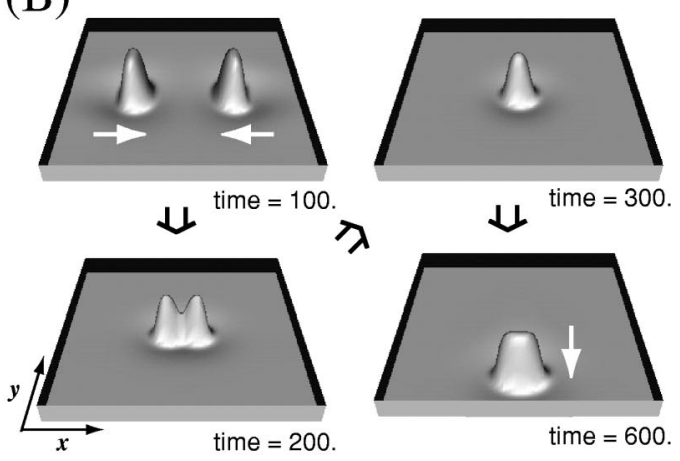

(C)

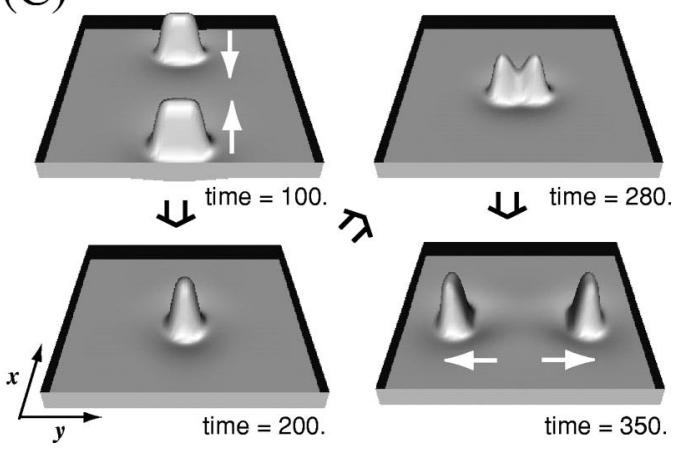

FIG. 18. (A), (B) Transition from reflection to fusion-drift spot process occurs at $\tau \approx 69.54853$ as $\tau$ is increased. (C) When $\tau$ is slightly larger than $\tau_{s} \approx 84.52735$, the splitting of the fused spot occurs. The gourd and fusion scattors intervene in these scattering processes. Note that the direction of two incoming pulses is parallel to that of two peaks of twin-horn scattor in (A), but orthogonal in (C). Only the $u$ component is shown here. The system size displayed here is $0.5 \times 0.5$.

stance, the first real positive eigenvalue of the gourd pattern is much larger than the remaining unstable ones and the associated eigenform is axisymmetric (Fig. 19). Therefore when we add a small perturbation of this eigenform to the gourd scattor, the output, depending on its sign of the perturbation, is either like Fig. 21(A), i.e., the middle part of the gourd rises and changes into a circular shape, or like Fig. 21(B), i.e., the depth of the valley of the gourd becomes deeper and splits into two propagating spots. This clarifies the distinction between Figs. 18(A) and 18(B). Suppose two spots fused into a single spot as in Fig. 18(B) $t=300$, then the orbital behavior is guided by the outputs from the fused scattor in Fig. 22, namely it starts to move in one direction because fused scattor has only drift instabilities. Note that the direction of the drift is difficult to predict, since it de- 
(A)
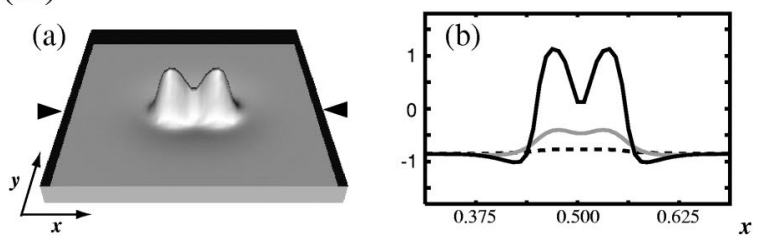

(B)
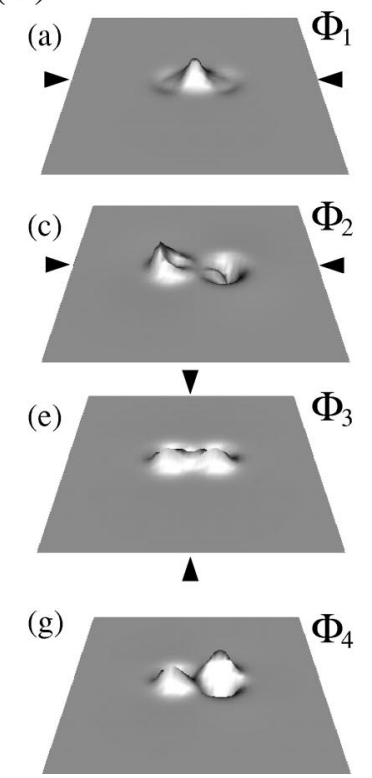
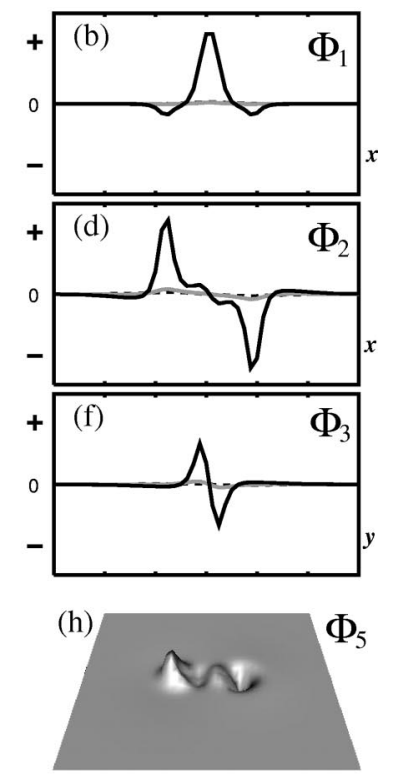

FIG. 19. (A) Gourd scattor when $\tau \approx 69.54853$. (a) Only the $u$ component is shown here and the profiles along the $x$ axis are depicted in (b). The solid, gray, and broken lines indicate $u, v$, and $w$ components, respectively. (B) The five unstable eigenvalues are $\lambda_{1}=0.6275>\lambda_{2}=0.0959>\lambda_{3}=0.0601$ $>\lambda_{4}=0.0277>\lambda_{5}=0.0195$. The associated eigenfunctions $\Phi_{i} \quad(i$ $=1, \ldots, 5)$ and the corresponding profiles are shown in (a)-(h).

pends on a tiny fluctuation of the initial data and so on.

The input-output relation undergoes the second qualitative change around $\tau_{s} \approx 84.52735$ from one-spot-emission to two-spot-emission [see Figs. 18(B) and 18(C)]. Two spots merge into a single spot [Fig. 18(C) $t=200$ ], however the orbit almost immediately changes into the twin-horn shape [Fig. 18(C) $t=280$ ] and stays there for certain time, and finally splits into two propagating spots. The gourd scattor plays a similar role as in the previous case, however it should be noted that the direction of the line connecting two humps of the gourd is orthogonal to that of incoming spots, which makes a contrast with the repulsion case [Fig. 18(A)]. This observation may give an insight about the "asymmetry" of the angles of incidence and reflection in dissipative systems. ${ }^{34}$ In view of Fig. 18(C), we see that the direction of two outgoing spots is orthogonal to that of incoming ones, namely the angle of incidence (respectively, reflection) is $\pi / 2$ (respectively, 0). Noting that the straight line connecting the tops of twin-horn scattor is parallel to the direction of outgoing spots, it is natural to see that the direction of the scattor, which breaks the $D^{\infty}$ symmetry (i.e., symmetry of the circle consisting of rotations and reflections), may cause the asymmetry of the above two angles. Intuitively the distributions of the inhibitors have longer tails due to the difference of diffusivities, hence outgoing spots prefer the orthogonal

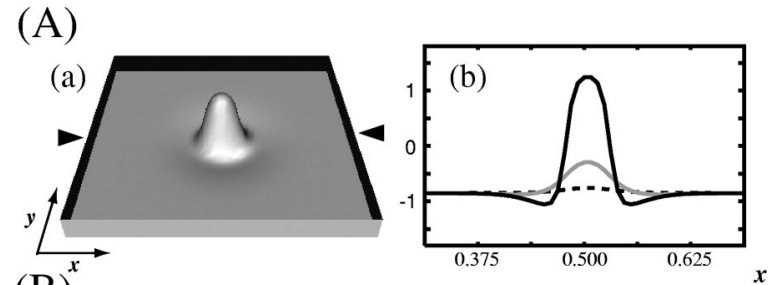

(B)

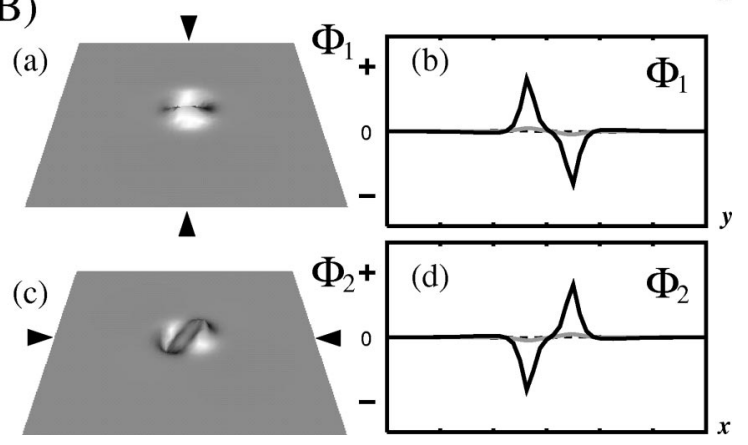

FIG. 20. (A) Fusion scattor when $\tau \approx 69.54853$. (a) Only the $u$ component is shown here and the profiles along the $x$ axis are depicted in (b). The solid, gray, and broken lines indicate $u, v$, and $w$ components, respectively. (B) The two unstable eigenvalues are $\lambda_{1}=\lambda_{2}=0.0921$. The associated eigenfunctions $\Phi_{1}$ and $\Phi_{2}$ and the corresponding profiles are shown in (a)-(d).

direction. It should be remarked that the asymmetry of two angles still persists for scattering slightly off the head-on collision by continuous dependence of orbital behaviors on initial conditions.
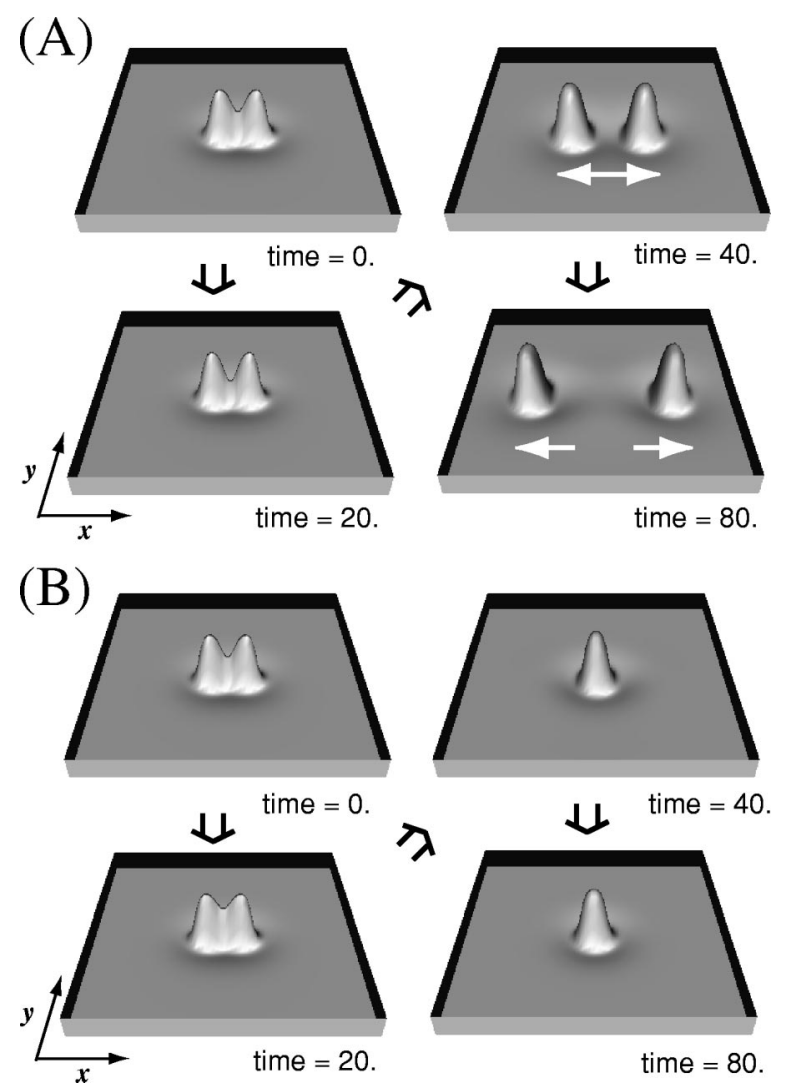

FIG. 21. Response of the gourd scattor by adding a small perturbation in the direction of $\Phi_{1}$ when $\tau \approx 69.548$ 53. (A) [respectively, (B)] Positive (respectively, negative) perturbation. These are consistent with the outputs in Figs. 18(A) and 18(B). 


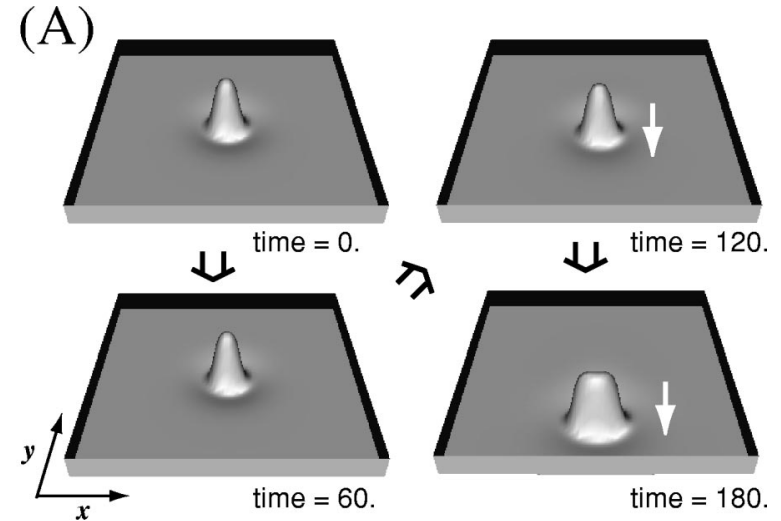

(B)

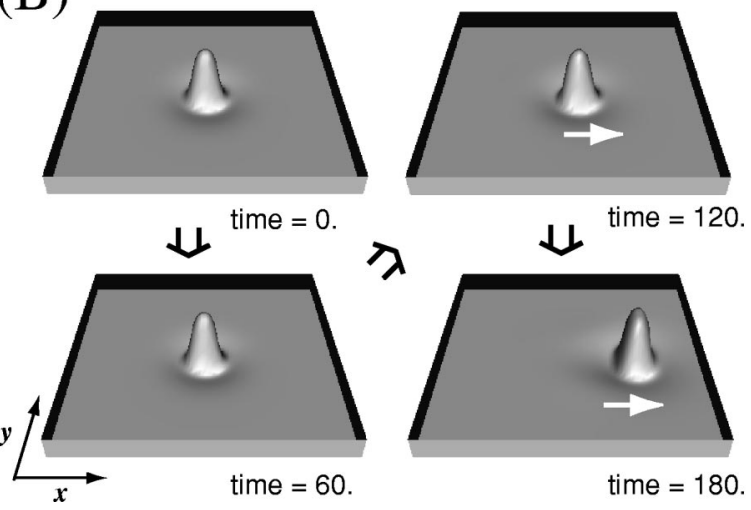

FIG. 22. (A) [respectively, (B)] Response of the fusion scattor by adding a small positive perturbation in the direction of $\Phi_{1}$ (respectively, $\Phi_{2}$ ) when $\tau \approx 69.54853$.

\section{CONCLUDING REMARKS}

Scattering phenomena among traveling pulses is studied. The input-output relation is changed depending on parameters and initial perturbations for dissipative systems. The origin of such a diversity may be reduced to the local dynamics around scattors; unstable steady or time-periodic solutions which control the flows nearby. The orbit approaches a scattor right after collision and is sorted out along one of the unstable directions of the scattor. It is not always true that the output is an ordered pattern like pulses or spots, in fact a spatiotemporal chaos is produced through scattering process for the GS model (2) in an appropriate parameter region. The outputs can be classified by looking at the outcome from the scattor along its unstable manifolds. Moreover the output can be predictable by using the information on the solution profile right after collision, scattors, and their unstable eigenforms. We illustrate by using several models in 1D and 2D that scattors in dissipative systems may be ubiquitous and play a pivotal role to understand the transient process of scattering in dissipative systems.

\section{ACKNOWLEDGMENT}

This work was supported by the Grant-in-Aid for Scientific Research (B)13440027 and for Exploratory Research 14654018.

${ }^{1}$ Y. Astrov and H.-G. Purwin, Phys. Lett. A 283, 349 (2001).

${ }^{2}$ M. Bode, A. W. Liehr, C. P. Schenk, and H.-G. Purwins, Physica D 161, 45 (2002).

${ }^{3}$ M. Bär, M. Eiswirth, H.-H. Rotermund, and G. Ertl, Phys. Rev. Lett. 69, 945 (1992).

${ }^{4}$ M. G. Zimmermann et al., Physica D 110, 92 (1997).

${ }^{5}$ A. von Oertzen, A. S. Mikhailov, H. H. Rotermund, and G. Ertl, J. Phys. Chem. B 102, 4966 (1998).

${ }^{6}$ P. de. Kepper, J. J. Perraud, B. Rudovics, and E. Dulos, Int. J. Bifurcation Chaos Appl. Sci. Eng. 4, 1215 (1994).

${ }^{7}$ K. J. Lee, W. D. McCormick, J. E. Pearson, and H. L. Swinney, Nature (London) 369, 215 (1994).

${ }^{8}$ V. Petrov, S. K. Scott, and K. Showalter, Philos. Trans. R. Soc. London, Ser. A 347, 631 (1994).

${ }^{9}$ J. E. Pearson, Science 216, 189 (1993).

${ }^{10}$ S.-I. Ei, M. Mimura, and M. Nagayama, Physica D 165, 176 (2002).

${ }^{11}$ M. Mimura and M. Nagayama, Chaos 7, 817 (1997).

${ }^{12}$ T. Ohta, J. Kiyose, and M. Mimura, J. Phys. Soc. Jpn. 66, 1551 (1997).

${ }^{13}$ T. Ohta, Physica D 151, 61 (2001).

${ }^{14}$ K. Krischer and A. Mikhailov, Phys. Rev. Lett. 73, 3165 (1994).

${ }^{15}$ S. Kawaguchi and M. Mimura, SIAM (Soc. Ind. Appl. Math.) J. Appl. Math. 59, 920 (1999).

${ }^{16}$ Y. Nishiura and D. Ueyama, Physica D 150, 137 (2001).

${ }^{17}$ Y. Hayase and T. Ohta, Phys. Rev. E 62, 5998 (2000).

${ }^{18}$ Y. Nishiura and D. Ueyama, Physica D 130, 73 (1999).

${ }^{19}$ Y. Nishiura, Far-from-Equilibrium Dynamics, Translations of Mathematical Monographs Vol. 209 (American Mathematical Society, Providence, 2002).

${ }^{20}$ M. Argentina, P. Coullet, and L. Mahadevan, Phys. Rev. Lett. 79, 2803 (1997).

${ }^{21}$ P. Coullet, J. Lega, B. Houchmanzadeh, and J. Lajzerowicz, Phys. Rev. Lett. 65, 1352 (1990).

${ }^{22}$ S. Sasa, T. Mizuguchi, and M. Sano, Europhys. Lett. 19, 593 (1992).

${ }^{23}$ T. Mizuguchi and S. Sasa, Prog. Theor. Phys. 89, 599 (1993).

${ }^{24}$ P. Coullet and K. Emilson, Physica D 61, 119 (1992).

${ }^{25}$ H. Sakaguchi, Prog. Theor. Phys. 88, 593 (1992).

${ }^{26}$ H. Chate, A. Pikovsky, and O. Rudzick, Physica D 131, 17 (1999).

${ }^{27}$ M. Sano, K. Sato, S. Nasuno, and H. Kokubo, Phys. Rev. A 46, 3540 (1992).

${ }^{28}$ E. J. Doedel, A. R. Champneys, T. F. Fairgrieve, Y. A. Kuznetsov, B. Sandstede, and X. Wang, AUTO97, http://cmvl.cs.concordia.ca/auto (1997).

${ }^{29}$ W. H. Press, B. P. Flannery, S. A. Teukolsky, and W. T. Vetterling, Numerical Recipes in $C$ (Cambridge University Press, Cambridge, 1988).

${ }^{30}$ Y. Nishiura, T. Teramoto, and K.-I. Ueda, Phys. Rev. E 67, 056210 (2003).

${ }^{31}$ P. Gray and S. K. Scott, Chem. Eng. Sci. 39, 1087 (1984).

${ }^{32}$ H.-G. Purwins, Y. Astrov, and I. Brauer, in The Fifth Experimental Chaos Conference, edited by M. Ding, W. L. Ditto, L. M. Pecora, and M. L. Spano (World Scientific, Singapore, 2001), pp. 3-13.

${ }^{33}$ C. P. Schenk, M. Or-Guil, M. Bode, and H.-G. Purwins, Phys. Rev. Lett. 78, 3781 (1997).

${ }^{34}$ M. Mimura (private communication). 